\title{
Efficient Non-Linear 3D Electrical Tomography and Finite Element Optimizations for Functional Source Imaging
}

\author{
Molinari M., Cox S.J., Blott B.H. and Daniell G.J. \\ University of Southampton, United Kingdom
}

\section{Introduction}

An essential factor in functional source imaging is the accurate knowledge of the conductivity distribution inside the body. Current models for electrophysiological forward and inverse problems use tabulated conductivity values obtained from experiments. These quantities usually show large standard deviations and an individual patient's conductivities might depart siginificantly from average values.

Electrical impedance tomography (EIT) provides a method of non-invasively obtaining information about in-vivo tissue conductivities by means of measuring surface potentials resulting from small injected currents. Fig. 1 shows how EIT-derived conductivities can be used in EEG reconstructions for a head slice. Previous work has been carried out to approximate tissue conductivities on simplified models with few variable conductivities, for example in [1] and [2]. Recent advances in EIT, however, show that it is theoretically possible to obtain good approximations of absolute conductivities for medical applications [3].

We address the influence of a finite element discretization on the solution of forward and inverse problem in tomographic reconstructions, discuss the implementation of an adaptive mesh refinement technique and present results from current density determination based on the improved conductivity image obtained by EIT.

\section{Method}

The electric potential $\phi(\mathbf{r})$ at location $\mathbf{r}$ inside the body with conductivity distribution $\sigma(\mathbf{r})$ is governed in EIT by the equation

$$
\nabla \cdot(\sigma(\mathbf{r}) \nabla \phi(\mathbf{r}))=0
$$

along with boundary conditions imposed by the employed electrode model.

The solution of this equation for $\sigma$ is based on the non-linear minimization of the functional

$$
\begin{aligned}
\Phi & =\sum_{i=1}^{M}\left(\frac{U_{i}(\sigma)-V_{i}}{\delta V_{i}}\right)^{2}+\lambda \int_{i m a g e}|\nabla \log \sigma|^{2} d^{3} \mathbf{r} \\
& =\chi^{2}+\lambda \Psi
\end{aligned}
$$

A good fit between measured, $\mathbf{V}$, and computed, $\mathbf{U}(\sigma)$, electrode voltages is reached when $\chi^{2} \approx M=$ the number of independent measurements made. $\delta \mathbf{V}$ contains the individual measurement errors and the

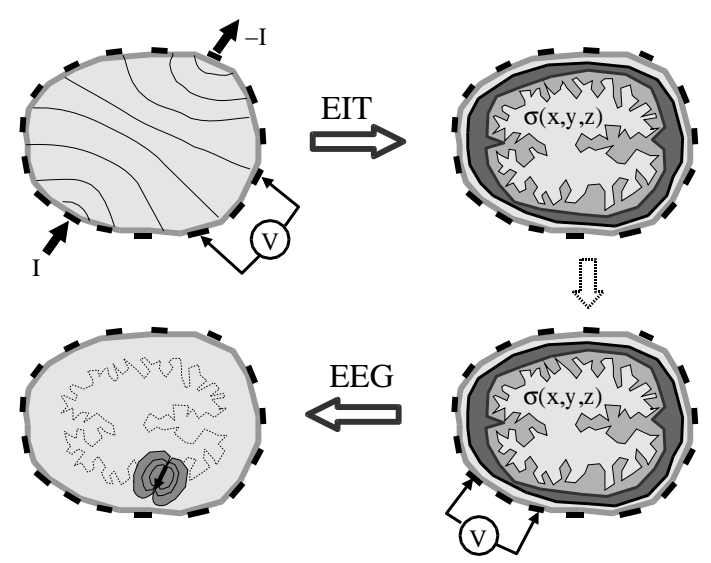

Figure 1: Inter-dependence of EIT and EEG reconstruction. The conductivity distribution obtained by EIT can be used for more accurate EEG inverse current dipole estimation.

image smoothness term $\Psi$ ensures a well-defined reconstruction. A more detailed dicussion of this functional and the determination of the parameter $\lambda$ can be found in [4] and [5].

For the numerical minimization of equation (2), we discretize the volume under investigation with tetrahedral finite elements with linear base functions for the potentials and a constant conductivity parameter for each element. The forward solution - which is required for the iterative minimization algorithm - can then be stated as linear system:

$$
\mathbf{U}=[\mathbf{Y}(D, \sigma)]^{-1} \mathbf{I} .
$$

I contains the currents injected through the electrodes and $Y$ denotes the admittance matrix. The finite element discretization $D$ significantly influences both the forward and the inverse solution in EIT. In the case of reconstructing neuro-active generators, we encounter a similarity: the formulation of the problem is given as $\mathrm{K}(D, \sigma) \mathbf{J}=\mathbf{V}$, where $K$ is the $D$ and $\sigma$ dependent lead field matrix and $J$ represents the sought current sources. To reduce the effects of the numerical discretization, we have developed a conjugate gradient based algorithm incoporating 3D auto-adaptive mesh refinement techniques. We have shown elsewhere that adaptive meshing is an optimal technique for the efficient solution of the forward problem [6] and neccessary to achieve the image resolution intrinsic in the measurement data [5].

For our studies, we use a 3D finite element head model with geometry derived from the Visual Human data 
set and apply a set of 32 point electrodes across the surface. Current injections through opposite located electrodes are made and the corresponding potential measurements are assumed to exhibit a measurement noise of $0.1 \%$.

The measurement simulation was carried out on a head model consisting of 92072 elements and containing regions with assigned conductivities of scalp $(0.44 \mathrm{~S} / \mathrm{m})$, skull $(0.02 \mathrm{~S} / \mathrm{m})$, CSF $(1.55 \mathrm{~S} / \mathrm{m})$ and brain $(0.25 \mathrm{~S} / \mathrm{m})$.

The initial mesh for the inverse solution contained 11509 elements which corresponds to a spatial resolution of approximately $3 \%$. As starting vector for the inverse solver, a homogenous $\sigma$ distribution was determined.

To compare the obtained results, we compute the average relative voxel error in the conductivity image by resampling the conductivities on a $100 \times 100 \times 100$ cube grid and by establishing the Frobenius norm between simulated $\sigma^{0}$ and reconstructed $\sigma$ :

$$
E=\sqrt{\frac{1}{100^{3}} \sum_{k=1}^{100^{3}}\left(\frac{\sigma_{k}-\sigma_{k}^{0}}{\sigma_{k}^{0}}\right)^{2}} .
$$

In addition, we investigated the effects of adaptive meshing on the reconstruction accuracy of a current source defined in a single finite element model in the brain. The relative error of this reconstruction was obtained similar to above by resampling the current density vector and evaluating a 3D Frobenius norm with the simulated current density.

\section{Results}

The numerical simulations show that the error of reconstructed tissue conductivities can be reduced from an initial $14 \%$ to approximately $4.2 \%$ when autoadaptive $3 \mathrm{D}$ mesh refinement techniques are employed to decrease the influence of the finite element discretization on the imaging process.

The effect of mesh refinement on the reconstruction of a simulated current density distribution are also evident: the error reduces by about a factor of 3 for all $\sigma$ estimates obtained from the EIT reconstructions. The lowest error is achieved for the best conductivity estimate. Fig. 2 shows the computed errors in conductivity and current density reconstruction.

\section{Discussion}

The presented results show that adaptive mesh refinement methods can improve tissue conductivity resolution in EIT significantly by reducing the effects of the finite element discretization on the reconstruction process. The same technique can be used to increase the spatial resolution in current source determination. Although EIT performs well in simulations, in-vivo measurements are the real challenge and have to prove in future work how applicable these methods are.

\section{Acknowledgments}

M. Molinari is grateful to $\operatorname{EPSRC}(\mathrm{UK})$ for funding.
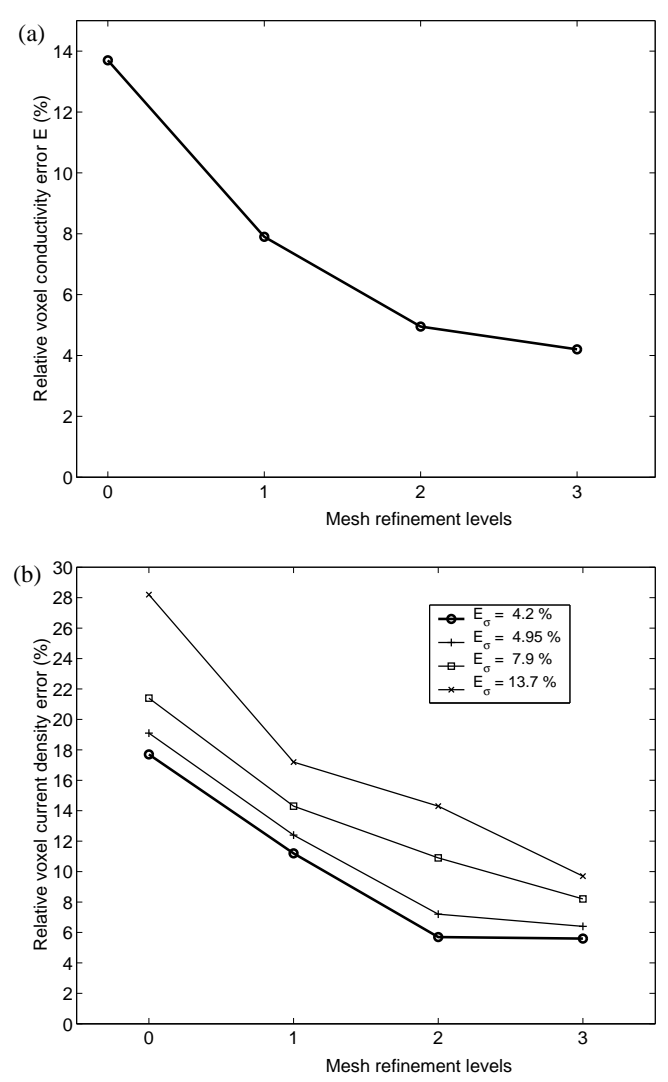

Figure 2: Average voxel error of (a) the EIT reconstructed tissue conductivity and (b) the localization of a simulated current source when the tissue conductivities from (a) are used.

\section{References}

[1] Ferree, T.C. and Tucker D.M. Development of High-resolution EEG Devices. IJBEM, 1999, 1(1): 4-10

[2] Starck, W.L., Sachse, F.B., and Doessel, O. Errors in reconstructed conductivity distributions due to noise in body surface potential measurements. In Proc. EMBEC 99, 1999, Vienna, Austria, 124-125

[3] Holder, D.S. (Ed), Biomedical Applications of EIT - Scientific Abstracts, $3^{\text {rd }}$ EPSRC Engineering Network Meeting, University College London, 46 April 2001, London

[4] Blott, B.H., Daniell, G.J., and Meeson, S. Nonlinear reconstruction constrained by image properties in electrical impedance tomography. Phys. Med. Biol., 1998, 43: 1215-1224

[5] Molinari, M., Blott, B.H., Cox, S.J., and Daniell, G.J. Optimal imaging with adaptive mesh refinement in electrical tomography. Submitted to Physiol. Meas. (June 2001)

[6] Molinari, M., Cox, S.J., Blott, B.H., and Daniell, G.J. Adaptive mesh refinement techniques for electrical impedance tomography. Physiol. Meas., 2001, 22(1): 91-96 